Advant ages of upright posi ti on i maging wi th medi um ener gy col I i nat or for sent i nel node I ymphosci nt i graphy i $n$ br east cancer pat i ent s

\begin{tabular}{|c|c|}
\hline 著者 & $\begin{array}{l}\text { Tsushi ma H r oyuki, Takayana Ter uhi ko, K zu } \\
\text { H r ot o, Yamanaga Takashi, Shi moni shi } \\
\text { Yoshi hi ro, Kosakai Kazuhi sa, Mur ai Nasam, } \\
\text { Onoguchi Masahi sa }\end{array}$ \\
\hline $\begin{array}{l}\text { j our nal or } \\
\text { publ i cat i on } t i t l e\end{array}$ & Annal s of Nucl ear Medi ci ne \\
\hline vol une & 21 \\
\hline number & 2 \\
\hline page $r$ ange & 123128 \\
\hline year & 2007-01- 01 \\
\hline URL & ht t p: //hdl . handl e. net /2297/3891 \\
\hline
\end{tabular}




\title{
Advantages of upright position imaging with medium-energy collimator for sentinel node lymphoscintigraphy in breast cancer patients
}

\author{
Hiroyuki Tsushima, $* * *$ Teruhiko Takayama, ${ }^{* *}$ Hiroto Kizu,** Takashi Yamanaga, ${ }^{*}$ \\ Yoshihiro Shimonishi, ${ }^{*}$ Kazuhisa KosaKai, ${ }^{*}$ Masami Murai* and Masahisa OnOGuchi** \\ *Department of Radiology, Osaka City University Hospital \\ **Department of Health Sciences, Graduate School of Medical Science, Kanazawa University
}

\begin{abstract}
Objective: To evaluate the advantage of upright position imaging with a medium-energy collimator for the detection of sentinel lymph node (SLN). Methods: Thirty-four patients with operable breast cancer underwent sentinel node lymphoscintigraphy with ${ }^{99 \mathrm{~m}} \mathrm{Tc}$-tin colloid. Images were obtained in 5 different positions and paired images from the same patient were compared using side-by-side interpretation. Images were compared in 3 groups: group 1 (anterior view); supine (SAV) vs. upright (UAV), group 2 (oblique view); supine (SOV) vs. upright (UOV), and group 3 (oblique view); modified supine (MOV) vs. UOV. Image quality was evaluated using a 3-grade scale of clear, faint, and equivocal depiction, and correlated to 3 parameters: distance from injection site to lymph node (hot node), counts in hot node, and image contrast. Parameters in group 1 were compared by classifying the primary tumor site into 4 subregions. Results: Image quality in all 3 groups was more enhanced on the image obtained in the upright position than that in the supine position. Obtaining images in an upright position increased the mean distances by $1.5-3.2 \mathrm{~cm}$, and mean contrasts were significantly increased by $0.13-0.31(\mathrm{p}<0.05)$. It was shown that image quality was more greatly affected by image contrast than by counts in the hot node. Image contrast of 0.5 seemed an appropriate threshold level for detection of the hot node. On comparison of tumor sites, the upper outer quadrant $(\mathrm{C})$ region of the 4 subregions demonstrated greater contrast enhancement on upright position images. Conclusion: Clinical images obtained in an upright position with a mediumenergy collimator were superior to those obtained in a supine position. Use of this procedure is recommended to enhance lymph node detection on sentinel node lymphoscintigraphy.
\end{abstract}

Key words: sentinel lymph node, breast cancer, lymphoscintigraphy, upright position, visual detection

\section{INTRODUCTION}

THERE ARE MANY REPORTS describing techniques to enhance sentinel lymph node (SLN) detection on sentinel node lymphoscintigraphy in breast cancer patients. ${ }^{1-10}$ Of many factors affecting the detection of SLN, patient positioning or choice of the best scintigraphic projection is one of the most important factors affecting satisfactory

Received August 1, 2006, revision accepted December 18, 2006.

For reprint contact: Hiroyuki Tsushima, C.N.M.T., Department of Radiology, Osaka City University Hospital, 1-5-7 Asahimachi, Abeno, Osaka 545-8586, JAPAN.

E-mail: tsushima@med.osaka-cu.ac.jp lymphoscintigraphic study. Sentinel node lymphoscintigraphy is usually obtained in a supine position, with the arm abducted completely to allow the head of the $\gamma$-camera to be placed as close as possible to the axilla. Some authors reported that addition of oblique view imaging to anterior view imaging in the conventional supine position improves the diagnostic accuracy. ${ }^{11-13}$ On imaging, a lead shield is used to cover the injection site with a high level of radioactivity. However, use of a lead shield has a disadvantage in that it may cover lymph nodes on images. We recently reported the usefulness of a medium-energy collimator for sentinel node lymphoscintigraphy in breast cancer patients. ${ }^{14,15}$ In this method, a medium-energy collimator used with an appropriate energy window setting decreases the occurrence of scattered radiation, thereby 


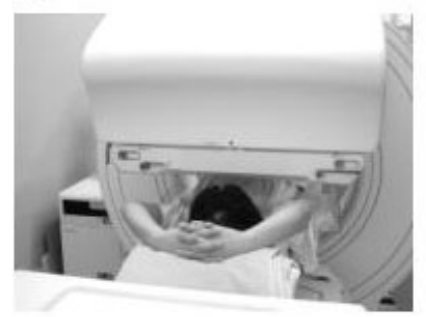

B

$\mathrm{C}$

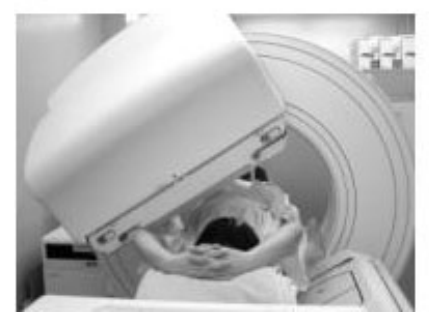

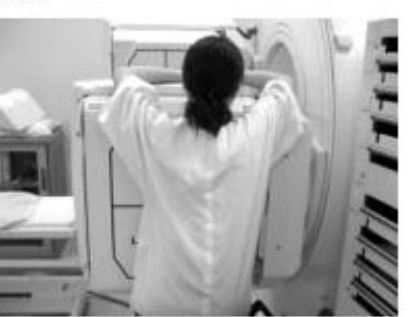

D

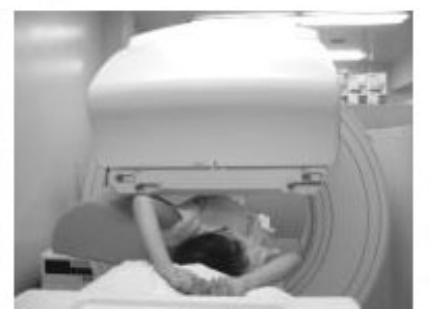

E

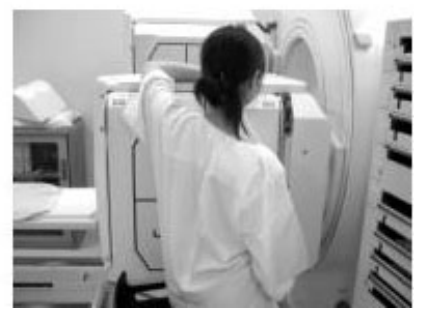

Fig. 1 Five imaging positions. (A) supine anterior view (SAV), (B) upright anterior view (UAV), (C) supine oblique view (SOV), (D) modified supine oblique view (MOV), and (E) upright oblique view (UOV).
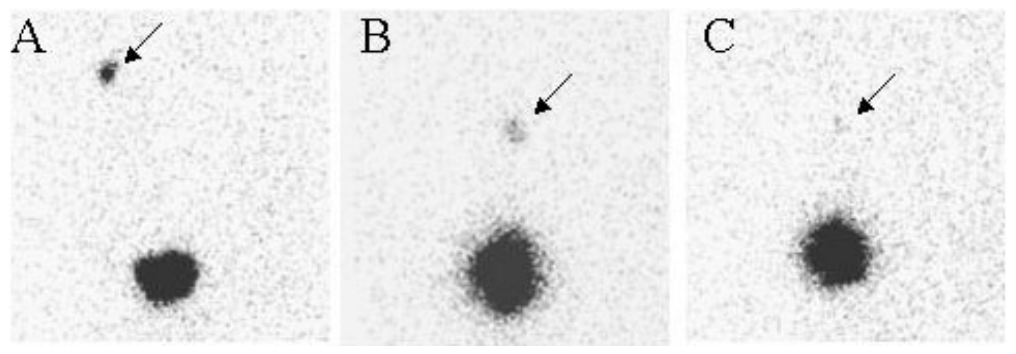

Fig. 2 Representative images of hot node depiction; (A) clear, (B) faint, and (C) equivocal. An arrow indicates the hot node.

enhancing image quality. Furthermore, this method enables imaging in an upright position because the procedure can be performed without a lead shield. Pierini et al. ${ }^{16}$ reported the benefits of imaging in an upright position, which allowed separation of the SLN and injection site. However, detailed quantitative estimation was not performed.

This study investigated the advantages of obtaining images in an upright position during sentinel node lymphoscintigraphy in breast cancer patients. Findings were assessed by quantitative evaluation of parameters, in addition to visual evaluation of images.

\section{MATERIALS AND METHODS}

\section{Patients}

This study comprised 34 patients (mean \pm SD age, $53.2 \pm$ $10.8 \mathrm{y}$; range, 35-74 y) with operable breast cancer. All patients underwent various examinations including ultrasonography, mammography, computed tomography, and bone scintigraphy to accurately locate the primary tumor and exclude remote metastasis. Tumor sizes ranged from 0.6 to $3 \mathrm{~cm}$ (mean \pm SD diameter, $1.64 \pm 0.78 \mathrm{~cm}$ ). Before surgery, 22 and 12 patients were diagnosed as having Stage I $(<2 \mathrm{~cm}$ in diameter) and Stage IIA $(2-3 \mathrm{~cm})$ breast cancer, respectively. Primary tumor was located in the upper inner quadrant (A), lower inner quadrant (B), upper outer quadrant (C), and lower outer quadrant (D) regions in $10,4,12$, and 8 patients, respectively. Histopathological diagnosis was established using needle biopsy and surgery specimens. Before examination, written informed consent was obtained from each patient.

\section{Imaging}

Imaging was performed with a dual-head $\gamma$-camera system (FORTE; ADAC, Milpitas, CA, USA) equipped with a medium-energy general purpose (MEGP) collimator. Tracer ( $3 \mathrm{ml}, 40 \mathrm{MBq}{ }^{99 \mathrm{~m}} \mathrm{Tc}$-tin colloid) was divided into 4 aliquots of $0.7 \mathrm{ml}$ and was injected at 4 peritumoral sites corresponding to the $12,3,6$, and 9 o'clock positions and at the same depth as the center of tumor. All injections were performed by one physician to avoid differences in 
injection technique. Approximately $4 \mathrm{hrs}$ after the injection, a static image was obtained with a matrix of $256 \times$ 256 (pixel $=2.36 \mathrm{~mm}$ ) for $250 \mathrm{sec}$. To evaluate the effects of imaging positions on hot node identification, images were obtained in 5 positions (Fig. 1): (A) supine anterior view (SAV), (B) upright anterior view (UAV), (C) supine oblique view (SOV), (D) modified supine oblique view (MOV), and (E) upright oblique view (UOV). As shown in Figure 1, the patient's arms were raised toward the head in each position. Each patient underwent imaging in at least 2 positions.

\section{Image interpretation}

Images were interpreted side-by-side on a workstation (Pegasys, ADAC) by consensus of 3 radiological technologists with more than $10 \mathrm{yrs}$ experience in nuclear medicine techniques. As shown in Figure 2, image quality on hot node was evaluated using 3 grades (clear, faint, and equivocal depiction) and was compared in 3 groups: group $1(\mathrm{n}=50)$; SAV vs. UAV in 34 patients, group 2 ( $\mathrm{n}$ $=15)$; SOV vs. UOV in 9 patients, and group $3(n=27)$; UOV vs. MOV in 18 patients.

\section{Data analysis}

The following 3 parameters were quantitatively evaluated: distance from the center of the injection site to hot node, counts in hot node, and image contrast. Counts were obtained from the value in the region of interest (ROI) of $4 \times 4$ pixels placed on a hot node image. Net counts in hot node were calculated by subtracting background counts from the counts in hot node. Image contrast was calculated using the following formula:

$$
\mathrm{C}=(\mathrm{SLN}-\mathrm{BG}) /(\mathrm{SLN}+\mathrm{BG})
$$

Eq. 1

where $\mathrm{C}$ represents image contrast, SLN represents the counts in the hot node ROI, and BG represents the counts in the background. As the background ROI, $4 \times 4$ pixels were placed near the hot node on the image. The distance from injection site to background ROI was similar to that from injection site to the hot node. Image quality was correlated to the values of parameters. In addition, classifying primary tumor sites into the 4 subregions of A, B, C, and $\mathrm{D}$, distance and contrast were obtained in Group 1.

\section{Statistical analysis}

Statistical significance was calculated with Wilcoxon's signed rank sum test and student t-test. Differences were considered significant at $\mathrm{p}<0.05$.

\section{RESULTS}

\section{Hot node detection with visual image interpretation}

A total of 50 hot nodes were identified in 34 patients. Sentinel node scintigraphy visualized 1,2 , and 3 hot nodes in 21,10 , and 3 patients, respectively, which were
Table 1 Comparison of image quality between 2 positions

A: Group 1

\begin{tabular}{lrccr}
\hline \multirow{2}{*}{ SAV } & \multicolumn{3}{c}{ UAV } & total \\
\cline { 2 - 4 } & clear & faint & equivocal & \\
\hline clear & 17 & 1 & 0 & 18 \\
faint & 6 & 0 & 0 & 6 \\
equivocal & 14 & 11 & 1 & 26 \\
\hline \multicolumn{1}{c}{ total } & 37 & 12 & 1 & 50 \\
\hline
\end{tabular}

B: Group 2

\begin{tabular}{lcccc}
\hline \multirow{2}{*}{ SOV } & \multicolumn{3}{c}{ UOV } & \multirow{2}{*}{ total } \\
\cline { 2 - 4 } & clear & faint & equivocal & \\
\hline clear & 7 & 2 & 0 & 9 \\
faint & 2 & 1 & 0 & 3 \\
equivocal & 3 & 0 & 0 & 3 \\
\hline \multicolumn{1}{c}{ total } & 12 & 3 & 0 & 15 \\
\hline
\end{tabular}

C: Group 3

\begin{tabular}{lrccr}
\hline \multirow{2}{*}{ MOV } & \multicolumn{3}{c}{ UOV } & \multirow{2}{*}{ total } \\
\cline { 2 - 4 } & clear & faint & equivocal & \\
\hline clear & 17 & 0 & 0 & 17 \\
faint & 2 & 5 & 0 & 7 \\
equivocal & 1 & 2 & 0 & 3 \\
\hline \multicolumn{1}{c}{ total } & 20 & 7 & 0 & 27 \\
\hline
\end{tabular}

A (Group 1); SAV vs. UAV, B (Group 2); SOV vs. UOV, and C (Group 3); MOV vs. UOV.

Case1.
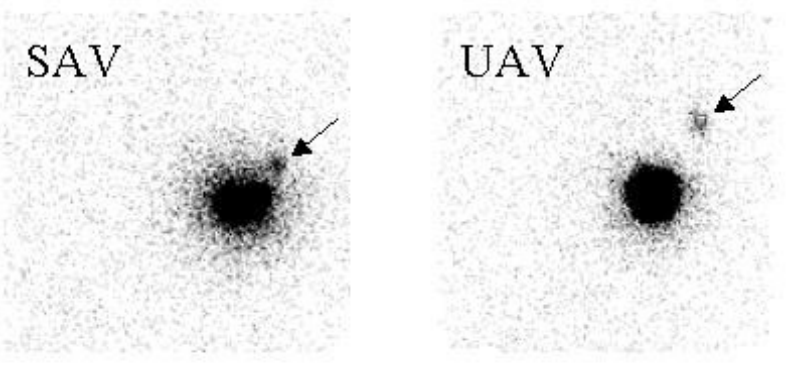

\section{Case2.}

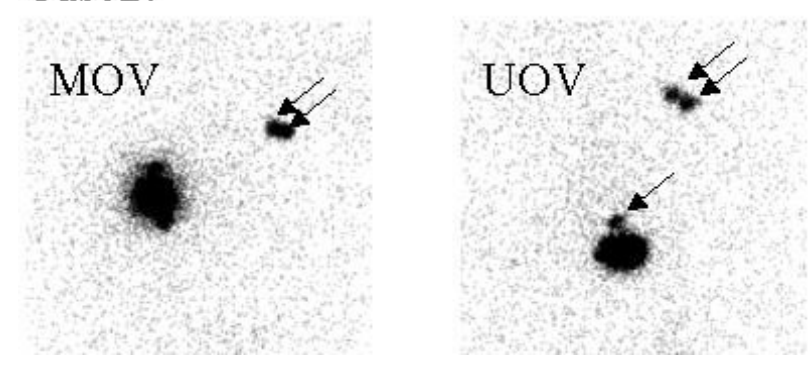

Fig. 3 Comparison of images in case 1 (SAV vs. UAV), and case 2 (MOV vs. UOV). Note that UAV image in case 1 depicted a hot node away from the injection site, and that UOV image in case 2 depicted the separation of 2 hot spots. 
A

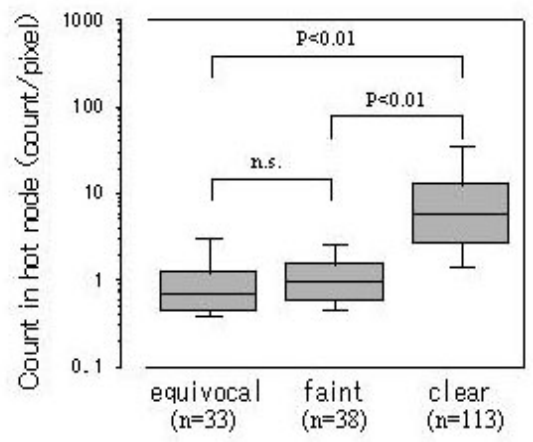

B

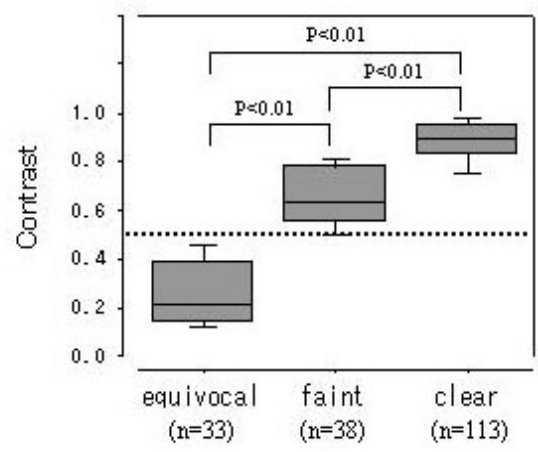

Fig. 4 Relationship between visual evaluation of images and counts in hot node (A), and contrast (B). Note the log scale on the longitudinal axis in (A). Dotted line indicates contrast of 0.5 .

confirmed by surgery. Table 1 shows a comparison of image quality in the 3 groups. In group 1 (SAV vs. UAV) in 34 patients, 37 hot nodes showed clear depiction on UAV images, compared with 18 hot nodes on SAV images. Furthermore, 17 (34\%) of 50 hot nodes on both images were concordantly interpreted as showing clear depiction. In group 2 (SOV vs. UOV) in 9 patients, 12 hot nodes showed clear depiction on UOV images, compared with 9 hot nodes on SOV images. Similarly, 7 (46.6\%) of 15 hot nodes on both images were concordantly interpreted as showing clear depiction. In group 3 (MOV vs. UOV) in 18 patients, 20 hot nodes showed clear depiction on UOV images, compared with 17 hot nodes on MOV images. Accordingly, 17 (62.9\%) of 27 hot nodes on both images were concordantly interpreted as showing clear depiction. Overall, it was shown that obtaining images in an upright position provided superior image quality compared to those in the supine position. Figure 3 shows a comparison of representative images in case 1 (group 1) and case 2 (group 3 ). Both cases showed marked improvement in the upright position.

\section{Distance from injection site to hot node}

Mean distances to hot nodes on images obtained in an upright position were $10.4 \pm 3.3 \mathrm{~cm}, 12.4 \pm 4.1 \mathrm{~cm}$, and $12.3 \pm 3.0 \mathrm{~cm}$ in groups 1,2 , and 3 , respectively, compared with $7.2 \pm 2.8 \mathrm{~cm}, 9.4 \pm 3.2 \mathrm{~cm}$, and $10.8 \pm 3.2 \mathrm{~cm}$ on those
Table 2 Comparison of distance and contrast in the 4 subregions in group 1

A

\begin{tabular}{lrrc}
\hline \multirow{2}{*}{ Region } & \multicolumn{3}{c}{ Distance $(\mathrm{cm})$} \\
\cline { 2 - 4 } & Supine & \multicolumn{1}{c}{ Upright } & Increment \\
\hline A $(\mathrm{n}=14)$ & $6.9 \pm 3.1$ & $9.0 \pm 3.1$ & $2.1 \pm 1.1(\mathrm{p}<0.01)$ \\
B $(\mathrm{n}=6)$ & $8.1 \pm 3.5$ & $11.8 \pm 3.5$ & $3.7 \pm 1.3(\mathrm{p}<0.05)$ \\
C $(\mathrm{n}=17)$ & $6.5 \pm 2.7$ & $9.5 \pm 1.6$ & $3.0 \pm 1.6(\mathrm{p}<0.01)$ \\
D $(\mathrm{n}=13)$ & $8.2 \pm 2.2$ & $12.5 \pm 3.9$ & $4.3 \pm 2.5(\mathrm{p}<0.01)$ \\
\hline
\end{tabular}

B

\begin{tabular}{lccc}
\hline \multirow{2}{*}{ Region } & \multicolumn{3}{c}{ Contrast } \\
\cline { 2 - 4 } & Supine & Upright & Increment \\
\hline A $(\mathrm{n}=14)$ & $0.51 \pm 0.34$ & $0.84 \pm 0.17$ & $0.33 \pm 0.26(\mathrm{p}<0.01)$ \\
B $(\mathrm{n}=6)$ & $0.53 \pm 0.34$ & $0.77 \pm 0.22$ & $0.24 \pm 0.21(\mathrm{p}<0.05)$ \\
C $(\mathrm{n}=17)$ & $0.46 \pm 0.31$ & $0.82 \pm 0.14$ & $0.36 \pm 0.24(\mathrm{p}<0.01)$ \\
D $(\mathrm{n}=13)$ & $0.60 \pm 0.23$ & $0.86 \pm 0.12$ & $0.25 \pm 0.20(\mathrm{p}<0.01)$ \\
\hline
\end{tabular}

obtained in a supine position, respectively. Clearly, obtaining images in an upright position significantly increased the distance by $3.2 \pm 1.9 \mathrm{~cm}, 3.0 \pm 1.4 \mathrm{~cm}$, and 1.5 $\pm 1.2 \mathrm{~cm}$ in Groups 1,2, and 3, respectively (respectively, $\mathrm{p}<0.01$ ).

\section{Counts in hot node and image contrast}

Figures 4 (A) and 4 (B) show the relationship between visual evaluation of images and counts (counts per pixel), and between visual evaluation of images and contrast, respectively, for 92 hot nodes with 2 views each. There was a significant difference in counts in hot node between clear and equivocal depiction, and between clear and faint depiction (respectively, $\mathrm{p}<0.01$ ), indicating higher counts in hot nodes with clear depiction. Similarly, there was a significant difference in image contrast among clear, faint, and equivocal depiction (respectively, $\mathrm{p}<0.01$ ), indicating markedly higher image contrast with clear depiction. As shown in Figure 4 (B), the line showing contrast of 0.5 discriminated equivocal depiction from faint and clear depiction.

Figure 5 shows a comparison of image contrast in 3 groups. The values in groups 1,2 , and 3 were $0.52 \pm 0.30$, $0.67 \pm 0.30$, and $0.73 \pm 0.24$ on supine position images, respectively, compared with $0.83 \pm 0.15,0.86 \pm 0.08$, and $0.86 \pm 0.11$ on upright position images, respectively. Significant differences were observed between supine and upright position images ( $\mathrm{p}<0.01$ for groups 1 and 3 , and $\mathrm{p}<0.02$ for group 2 , respectively). Consequently, images obtained in an upright position demonstrated improvement of contrast enhancement values by $0.31 \pm$ $0.23,0.19 \pm 0.27$, and $0.13 \pm 0.17$ in groups 1,2 , and 3 , respectively. Except for a few cases, contrast enhancement was improved on images obtained in an upright position.

Furthermore, from the results in Figure 4 (B) and 
A

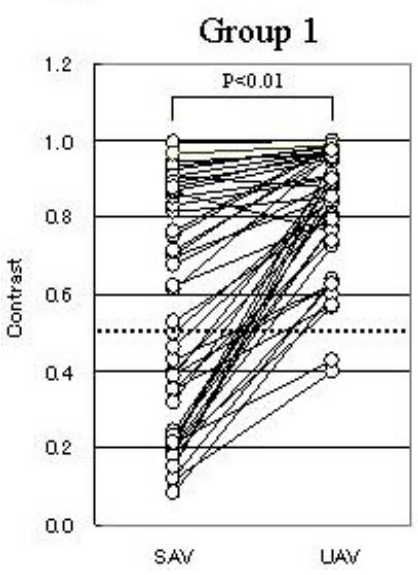

B

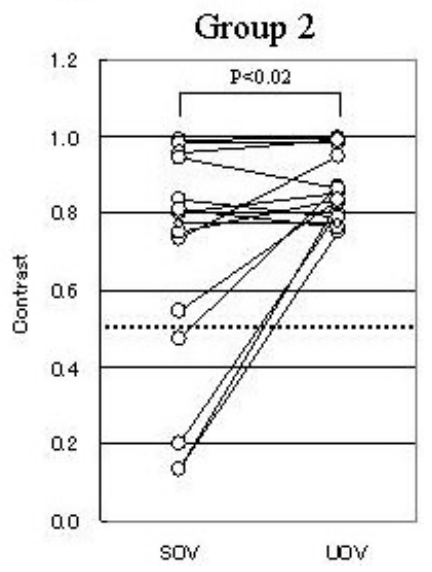

$\mathrm{C}$

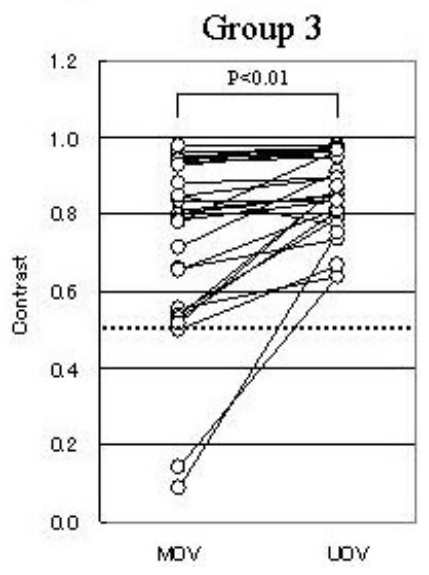

Fig. 5 Comparison of contrast between different imaging positions. Dotted line indicates image contrast of 0.5 .

Figure 5, we concluded that hot nodes could be detected with visual detection if a hot node had contrast of more than 0.5 on images obtained in an upright position.

\section{Values of parameters at different tumor sites}

As shown in Table 2, obtaining images in an upright position significantly increased distance (respectively, $\mathrm{p}<0.01$, or 0.05 ). By region, the increment of distance between supine and upright position was greater in order of D, B, C, and A. Similarly, images obtained in an upright position also demonstrated significantly increased contrast (respectively, $\mathrm{p}<0.01$, or 0.05 ). By region, increment of contrast between supine and upright position was greater in order of C, A, D, and B. That is, obtaining images in an upright position increased both distance and contrast compared to those on images in a supine position.

\section{DISCUSSION}

Imaging position is one of the most important factors for detection of SLN. ${ }^{1-10}$ Koizumi et al. ${ }^{11}$ demonstrated enhancement of hot node detection by adding the anterior oblique $60^{\circ}$ view to the anterior oblique $30^{\circ}$ view for a tumor located at the upper lateral quadrant region. They suggested that multi-directional views contributed to depicting axillary nodes behind the injected radioactivity. Haigh et al. ${ }^{12}$ reported a useful imaging method using a modified oblique view of the axilla (MOVA) (For convenience, we used the abbreviation MOV instead of MOVA in this paper). They obtained images after elevating the ipsilateral shoulder to $45^{\circ}$ on a triangular foam wedge and raising the arm overhead. Consequently, identification of axillary SLNs was equivalent with MOVA and anterior view in $18(24 \%)$ of 75 patients, was better with MOVA in $20(26 \%)$ patients, and was achieved only with MOVA in $38(50 \%)$ patients. Ichihara et al. ${ }^{13}$ confirmed the usefulness of MOVA, and proposed the combined appli- cation of MOVA and anterior view because SLN detection with $\gamma$-probe during surgery is performed in a supine position.

In contrast, Pierini et al. ${ }^{16}$ compared the sensitivity of SLN detection by lymphoscintigraphy obtained in the upright and supine positions in 23 breast cancer patients. Consequently, images obtained in an upright position improved the number of lymph nodes detected or the image quality in 20 of the 23 patients. Therefore, they reported that lymphoscintigraphy appeared to be more sensitive when obtained in the upright rather than supine position.

We recently reported the usefulness of a new imaging method using a medium-energy collimator. ${ }^{14}$ This method decreases the occurrence of star-shaped artifacts and scattered radiation using a medium-energy collimator with an appropriate energy window setting, thereby yielding improved image quality. ${ }^{15}$ This method is suitable for imaging in an upright position because it can be performed without a lead shield. In the present study, we investigated the usefulness of obtaining images in an upright position in comparison with those obtained in a supine position. Consequently, images in an upright position demonstrated excellent quality, mainly due to the increase in image contrast and in the distance from injection site to SLN. As Pierini et al. ${ }^{16}$ reported, an advantage of the upright position is based on the fact that the radiopharmaceutical injection site moves to a more inferior location away from the $\mathrm{LN}$ basin. The increased distance is beneficial in separating the hot node from the injection site. Actually, our findings showed that mean distances in the upright position were increased by about $3 \mathrm{~cm}$ compared to those in the supine position, and by about $1.5 \mathrm{~cm}$ compared to MOV imaging. In particular, the increment of distance was prominent in the D subregion.

Image quality is greatly affected by scattered radiation 
because the injection site with a high level of radioactivity exists near the lymph node with a low level of radioactivity. Our result showed that contrast in the upright position images was significantly increased by $0.24-0.36$ compared to supine position images, and hot node detection was more greatly affected by image contrast than counts in hot node. As shown in Figure 4, clear depiction of hot node correlated with higher contrast. As shown in Figure 5 , obtaining images in an upright position significantly improved contrast compared to that of images obtained in a supine position; this was particularly marked on the anterior view. In addition, images obtained in an upright position demonstrated 0.13 greater contrast than MOV imaging. From our results in Figure 4 and Figure 5, we concluded that image contrast of 0.5 was a suitable threshold level for hot node detection. A value of 0.5 indicates that the counts in hot nodes are 3 times greater than the background counts. The detection rate obtained with the threshold level was almost coincident with the results of visual image interpretation and of Pierini's report.

Enhancement of image contrast was most marked in the $\mathrm{C}$ region of 4 subregions. The improvement of contrast in the $\mathrm{C}$ subregion is very important because breast cancers arise more frequently in that area.

In this study, we considered that the detection of SLN is important. Therefore, the upright position was more useful than the supine position. However, if the exact location of SLN is requested, it is recommended to add the imaging in the supine position to imaging in the upright position. Further study is needed to be performed on the difference of SLN detection between by lymphoscintigraphy and by $\gamma$-probing during surgery because the latter is performed in a supine position.

\section{CONCLUSIONS}

Obtaining images in an upright position with a mediumenergy collimator enhanced image quality in sentinel node lymphoscintigraphy. The superiority of this approach was confirmed by quantitative evaluation of the distance, the count in hot node, and image contrast, as well as by visual evaluation of images.

\section{ACKNOWLEDGMENTS}

The authors thank K. Okuyama, chief technologist, and all radiological technologists in Osaka City University Hospital for providing technical support. We are grateful to S. Shiomi, Prof., J. Kawabe, M.D., and K. Torii, M.D., nuclear medicine physicians, for advice. This study was supported by a medical research grant from Osaka City University.

\section{REFERENCES}

1. Glass EC, Essner R, Giuliano AE. Sentinel node localiza- tion in breast cancer. Semin Nucl Med 1999; 29: 57-68.

2. Mariani G, Moresco L, Viale G, Villa G, Bagnasco M, Canavese $\mathrm{G}$, et al. Radioguided sentinel lymph node biopsy in breast cancer surgery. J Nucl Med 2001; 42: 1198-1215.

3. Dunnwald LK, Mankoff DA, Byrd DR, Anderson BO, Moe RE, Yeung RS, et al. Technical aspects of sentinel node lymphoscintigraphy for breast cancer. J Nucl Med Technol 1999; 27: 106-111.

4. Krynyckyi BR, Chun H, Kim HH, Eskandar Y, Kim CK, Machac J. Factor effecting depiction rates of internal mammary sentinel nodes during lymphoscintigraphy. $\mathrm{J} \mathrm{Nucl}$ Med 2003; 44: 1387-1393.

5. Krynyckyi BR, Kim CK, Goyenechea MR, Chan PT, Zhang ZY, Machac J. Clinical breast lymphoscintigraphy: optimal techniques for performing studies, image atlas, and analysis of images. Radiographics 2004; 24: 121-145.

6. Krynyckyi BR, Sata S, Zolty I, Kim CK, Knesaurek K. Reducing exposure from ${ }^{57} \mathrm{Co}$ sources during breast lymphoscintigraphy by optimizing energy windows and other suggested enhancements of acquisition and the display of images. J Nucl Med Technol 2004; 32: 198-205.

7. Chakera AH, Friis E, Hesse U, Al-Suliman N, Zerahn B, Hesse B. Factors of importance for scintigraphic nonvisualisation of sentinel nodes in breast cancer. Eur J Nucl Med Mol Imaging 2005; 32: 286-293.

8. Chen YW, Lai YC, Hsu CC, Hou MF. Enhanced sentinel lymphoscintigraphic mapping in breast tumor using graded shield technique. Kaohsiung J Med Sci 2003; 19: 339-343.

9. Veronesi U, Paganelli G, Galimberti V, Viale G, Zurrida S, Bedoni M. Sentinel-node biopsy to avoid axillary dissection in breast cancer with clinically negative lymph nodes. Lancet 1997; 349: 1864-1867.

10. Uren RF, Howman-Giles R, Renwick SB, Gillett D. Lymphatic mapping of the breast: locating the sentinel lymph nodes. World J Surg 2001; 25: 789-793.

11. Koizumi M, Nomura E, Yamada Y, Takiguchi T, Ishii M, Yamashita T, et al. Improved detection of axillary hot nodes in lymphoscintigraphy in breast cancer located in the upper lateral quadrant with additional projection imaging. Ann Nucl Med 2004; 18: 707-710.

12. Haigh PI, Hansen NM, Giuliano AE, Edwards GK, Ye W, Glass EC. Factors affecting sentinel node localization during preoperative breast lymphoscintigraphy. $\mathrm{J} \mathrm{Nucl} \mathrm{Med}$ 2000; 41: 1682-1688.

13. Ichihara H, Kinoshita F, Hiyoshi K, Beppu T, Fujigasaki K. Usefulness of imaging posture using modified oblique view of the axilla (MOVA) for sentinel lymph node scintigraphy in patients with breast cancer. Nippon Hoshasen Gijutsu Gakkai Zasshi 2003; 59: 765-770 (Japanese).

14. Tsushima H, Takayama T, Yamanaga T, Kizu H, Shimonishi Y, Kosakai K, et al. Usefulness of medium-energy collimator for sentinel node lymphoscintigraphy imaging in breast cancer patients. J Nucl Med Technology 2006; 34: 153-159.

15. Tsushima H, Yamanaga T, Shimonishi Y, Ochi H. Usefulness of imaging method without using lead plate for sentinel lymph node scintigraphy. KAKU IGAKU (Jpn J Nucl Med) 2002; 39: 161-169 (Japanese).

16. Pierini A, Dworkin HJ. Is the upright position more sensitive than the supine position in breast cancer sentinel node lymphoscintigraphy? Clin Nucl Med 2001; 26: 823-825. 